Supporting Information to accompany:

Optimization of Particle Transfers in the Gibbs Ensemble for Systems With Strong and Directional Interactions Using CBMC, $\mathrm{CFCMC}$, and $\mathrm{CB} / \mathrm{CFCMC}$

Ariana Torres-Knoop ${ }^{1 *}$, Nicholas C. Burtch ${ }^{2}$, Ali Poursaedesfahani ${ }^{3}$, Sayee Prasaad Balaji ${ }^{3}$, Ramses Kools ${ }^{1}$, Florent X. Smit ${ }^{1}$, Krista S. Walton ${ }^{2}$, Thijs J.H. Vlugt ${ }^{3}$, David Dubbeldam ${ }^{1}$

${ }^{1}$ Van 't Hoff Institute for Molecular Sciences, University of Amsterdam, Science Park 904, 1098XH Amsterdam, The Netherlands

${ }^{2}$ Georgia Institute of Technology, School of Chemical \& Biomolecular Engineering 778 Atlantic Drive, N.W. Atlanta, GA 30332

${ }^{3}$ Delft University of Technology, Process \& Energy Department, Leeghwaterstraat 39, 2628CB Delft, The Netherlands

*A.TorresKnoop@uva.nl 


\section{Acceptance probability and efficiency of SPCFw}

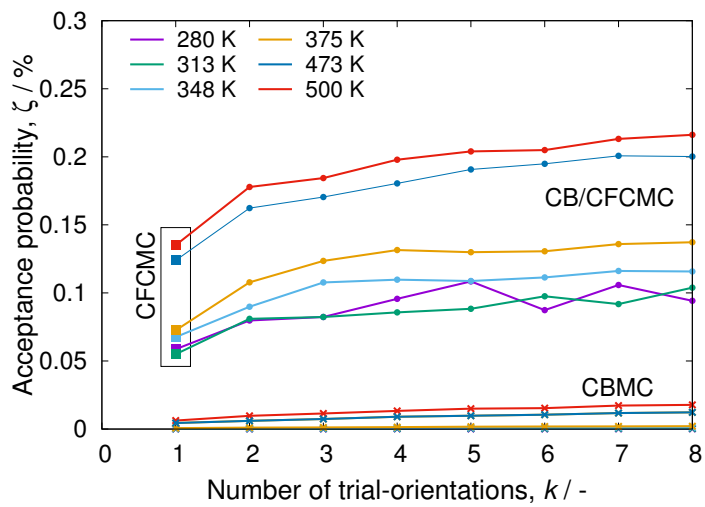

(a)

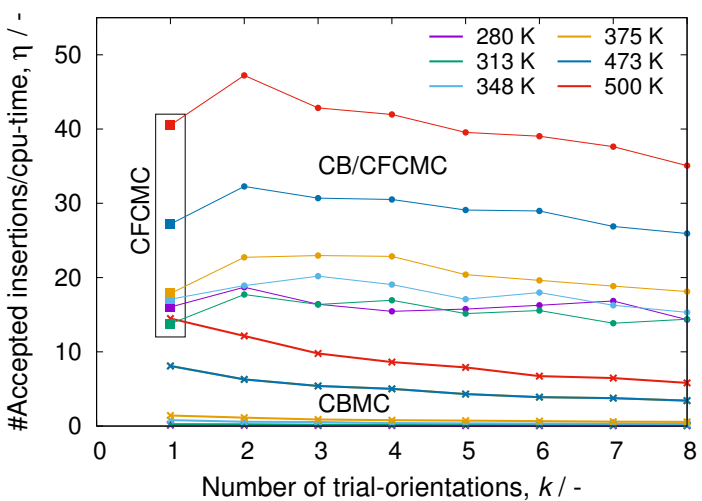

(b)

Figure S1: (a) Acceptance probability and (b) efficiency of the exchange trial move for Gibbs ensemble simulations using CBMC, CFCMC, CB/CFCMC algorithms and the SPCFw water model. Each color corresponds to a different temperature. The closed circles are the results obtained using $C B M C$, the crosses the results obtained with $C B / C F C M C$ and the closed squares the results obtained with CFCMC. The two methods using the expanded ensemble approach greatly improve the acceptance probability and efficiency. RASPA-2.0 software and an Intel CPU were used for this simulations.

\section{Modified biasing factors and probability distribution of $\lambda$ for SPC water}

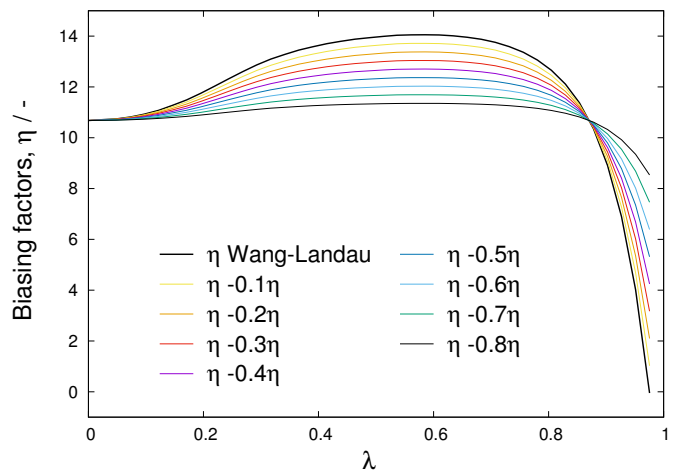

(a)

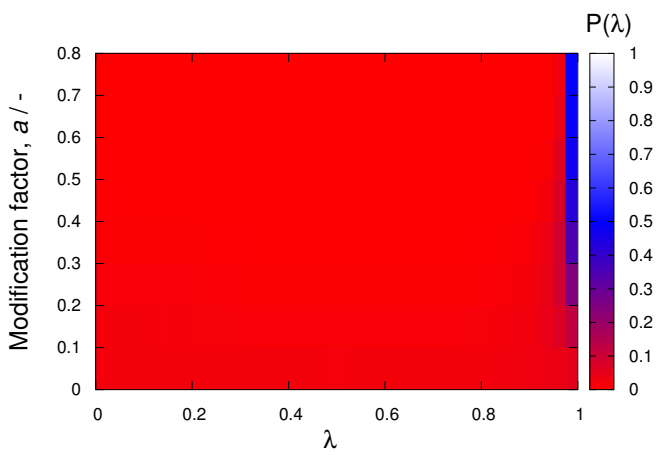

(b)

Figure S2: (a) Biasing factors (in units of $k_{B} T$ ) after adding $-a^{*} \eta$ to the original biasing factors (obtained during the equilibration using Wang-Landau), with a varying from 0 to 0.8. (b) Obtained probaility distribution of $\lambda$ with the modified biasing factors (Figure S2a). With an increasing modification factor, the coupling parameter gets 'more stuck' in values close to $\lambda=1$. 


\section{Probability distribution of $\lambda$ as a function of temperature for the water models}

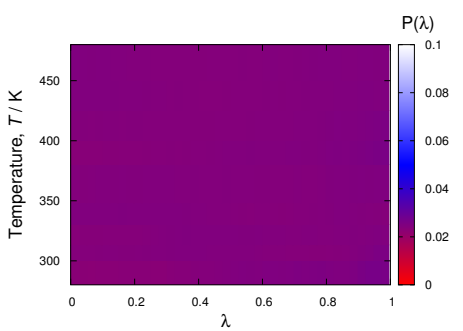

(a) SPC

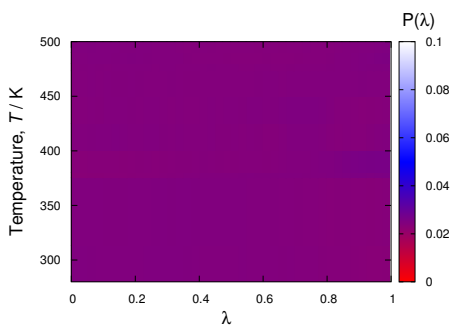

(b) $\mathrm{SPCFw}$

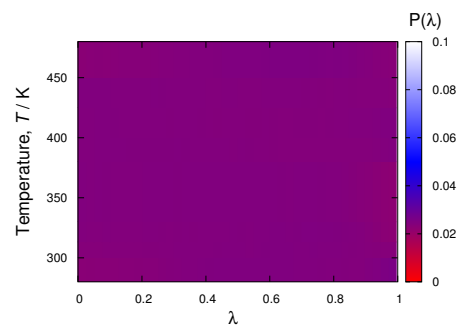

(c) Tip5p-Ew

Figure S3: Probability distribution of $\lambda$ obtained after including the biasing factors in the simulations as a function of temperature for the different water models using $C B / C F C M C$. A uniform color indicates that the $\lambda$-space is uniformly sampled.

\section{Biasing factors and probability distribution of $\lambda$ for DMF}
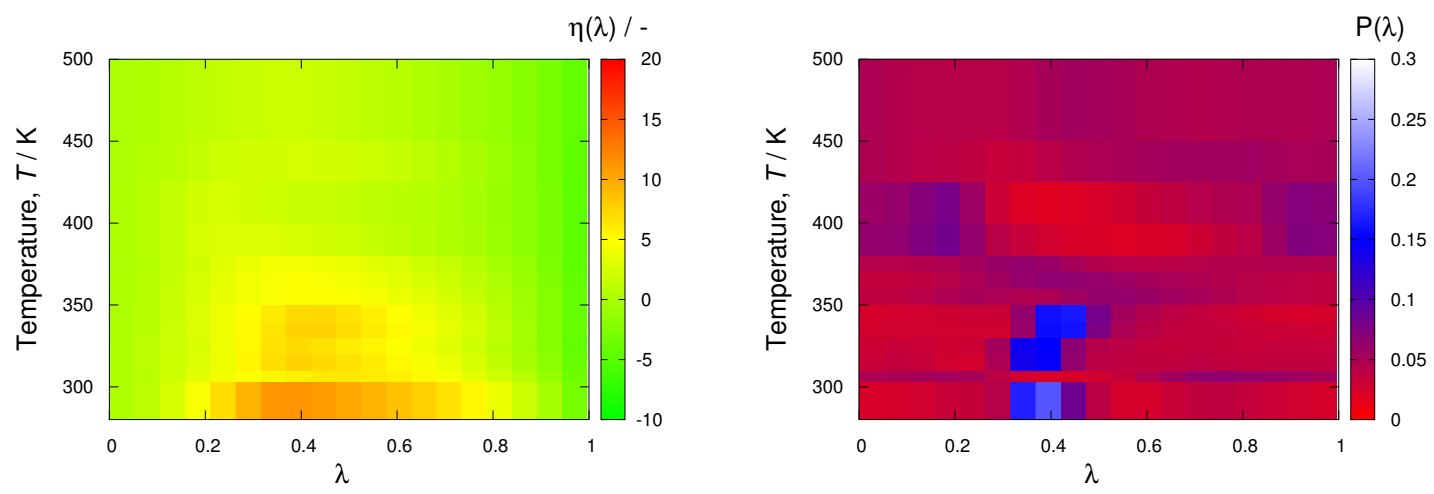

Figure S4: (a) Biasing factors (in units of $k_{B} T$ ) and (b) obtained probability distribution of $\lambda$ after including the biasing factors in the simulations as a function of temperature for $N, N$ - dimethylformamide (DMF). There appears to be a minimum around $\lambda=0.4$. 


\section{$5 \quad$ Biasing factors and probability distribution of $\lambda$ for SPC water adsorption in Zn-DMOF-TM after 500000 equilibration cycles}

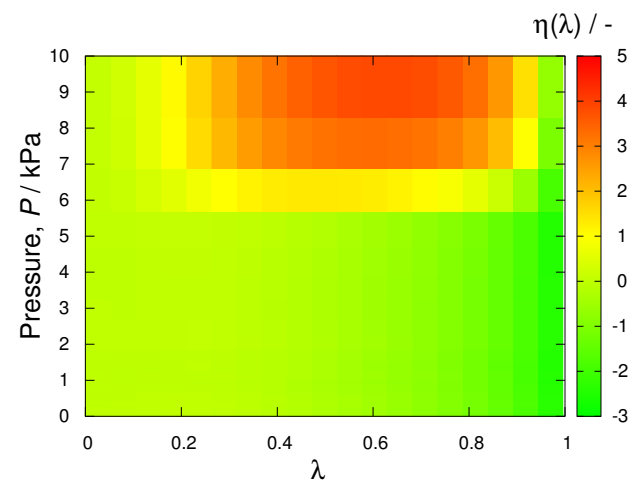

(a)

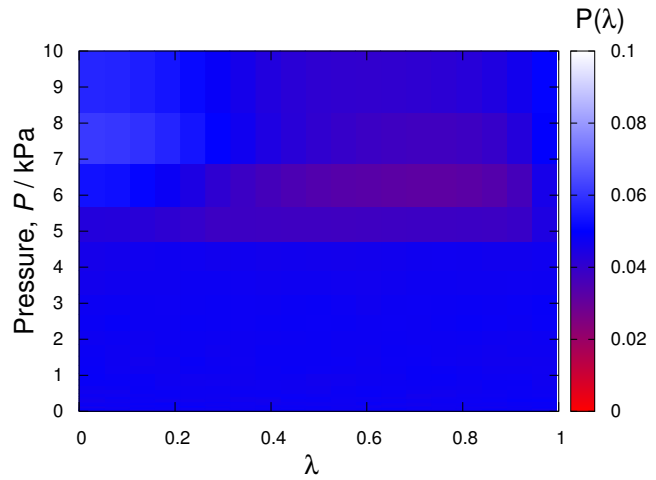

(b)

Figure S5: (a) Biasing factors (in units of $k_{B} T$ ) and (b) obtained probability distribution of $\lambda$ after including the biasing factors in the simulation of water adsorption in Zn-DMOF-TM at $300 \mathrm{~K}$.

\section{Longer simulations of two pressure points in the adsorption isotherm of water in Zn-DMOF-TM at $300 \mathrm{~K}$}

\begin{tabular}{ccc} 
Isotherm point $\mathrm{kPa}$ & Initial loading $[\mathrm{mol} / \mathrm{kg}]$ & Loading after more cycles $[\mathrm{mol} / \mathrm{kg}]$ \\
\hline 4.75 & $0.85+/-0.39$ & $2.59+/-1.65$ \\
5.7 & $7.93+/-5.75$ & $20.16+/-4.15$ \\
\hline
\end{tabular}

Table S1: Water loading in Zn-DMOF-TM after running the CB/CFCMC simulations for 10000 more equilibration cycles and 100000 more production cycles. The error was computed by dividing the production time in five blocks and computing the standard deviation of the block averages top the mean value.

\section{Simulations with one and two water molecules per unit cell using CBMC}

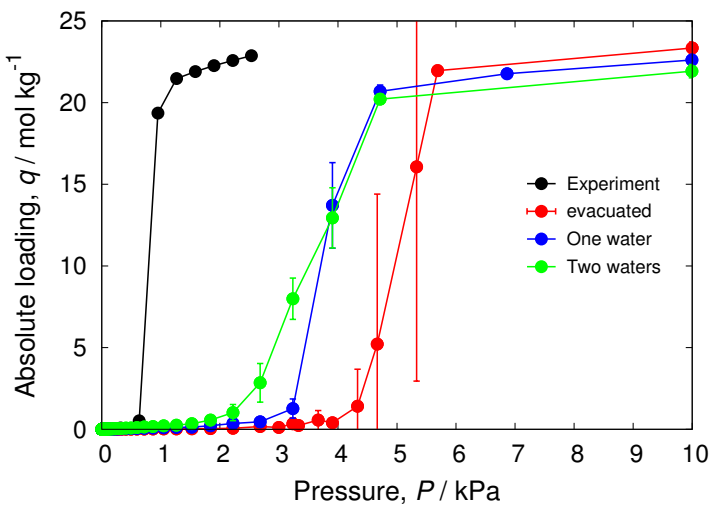

Figure S6: Effect of adding one and two preloaded water molecules in Zn-DMOF-TM structure on the adsorption isotherm of water at $300 \mathrm{~K}$ using the CBMC method. 